\title{
Efeitos da substituição parcial do milho na dieta pelo resíduo de panificação sobre o desempenho de novilhos da raça Holandesa ${ }^{(1)}$
}

\author{
Roberta Passini(2), Aleksandrs Spers ${ }^{(3)}$ e Carlos de Sousa Lucci( ${ }^{(3)}$
}

Resumo - Foi estudada a adição de resíduo de panificação (RP) em substituição ao milho, na dieta de novilhos Holandeses. Foram aplicados quatro tratamentos, correspondendo, respectivamente, à adição de $0 \%, 10 \%, 20 \%$ e $30 \%$ de RP na mistura de concentrados, em substituição ao milho. A alimentação fornecida foi ração completa, peletizada, contendo $30 \%$ de feno de Coast-cross (Cynodon dactylon L.) como volumoso. O delineamento experimental foi o de blocos casualizados, com quatro tratamentos e cinco repetições, para um total de 20 animais. Foram avaliados o consumo de matéria seca, conversão alimentar, ganho de peso, perímetro torácico e altura da cernelha. Avaliou-se a incidência de diarréia, por meio de observação diária das fezes. O experimento iniciou quando os animais atingiram $90 \mathrm{~kg}$ de peso vivo médio, e durou 120 dias. Os resultados não mostraram diferença estatística significativa entre os tratamentos em relação aos parâmetros estudados. A adição do RP causou redução de $3,74 \%$, $7,44 \%$ e $10,90 \%$ no custo de alimentação, respectivamente nos níveis $10 \%, 20 \%$ e $30 \%$ de RP, em comparação com a dieta-controle. O RP é uma fonte alternativa viável para a alimentação e redução dos custos de criação dos novilhos.

Termos para indexação: nutrição animal, ruminante, consumo alimentar, ganho de peso, custos de produção.

Effects of partial replacement of corn by bakery waste in the diet on performance of Holstein steers

\begin{abstract}
The effects of addition of bakery waste (BW) replacing corn in the diet of Holstein steers were studied. Four treatments, respectively to the addition of $0 \%, 10 \%, 20 \%$ and $30 \%$ of bakery waste were applied in the concentrated mixture for corn replacement. Animals were fed with complete mixtures pelleted ration, with $30 \%$ of Coast-cross hay (Cynodon dactylon L.) as roughage. The experimental design was in randomized blocks, with four treatments and five repetitions, totalizing 20 animals. The performance was evaluated by intake and feed conversion, weight gain, heart-girth and size. Diarrhea incidence was evaluated through daily observation of faeces. The experiment began when calves reached $90 \mathrm{~kg}$ of life weight, and lasted for 120 days. The results did not show statistically significant differences among treatments concerning weight gain, dry matter intake, feed conversion, heart-girth, height at whiter and diarrhea incidence. The addition of bakery waste caused feed costs reduction of $3.74 \%, 7.44 \%$ and $10.90 \%$ respectively for $10 \%, 20 \%$ and $30 \%$ of bakery waste, in comparison to the control diet $(0 \%)$. So, bakery waste is an alternative source for feeding and for reduction of costs of growing steers, in comparison to corn.
\end{abstract}

Index terms: animal nutrition, ruminants, feed intake, weight gain, production costs.

(1) Aceito para publicação em 17 de julho de 2000.

Extraído da dissertação de mestrado, apresentada pelo primeiro autor à Faculdade de Medicina Veterinária e Zootecnia (FMVZ), Universidade de São Paulo (USP), Pirassununga, SP. Trabalho financiado pela FAPESP.

(2)Universidade Estadual Paulista (UNESP), FMVZ, Dep. de Melhoramento e Nutrição Animal, Caixa Postal 560 CEP 18618-000, Botucatu, SP. E-mail: rpassini@bol.com.br

(3)USP, FMVZ, Dep. de Nutrição e Produção Animal, Caixa Postal 23, CEP 13630-000 Pirassununga, SP. E-mail: spers@usp.br

\section{Introdução}

Há considerável quantidade de resíduos de panificação disponíveis para utilização na alimentação animal, incluindo-se nestes as sobras de bolos, pães, biscoitos doces e salgados, produtos não comercializados ou que ultrapassaram o prazo de validade, além das perdas por quebras, excesso ou falta de cozimento durante o processamento (Wallace, 1965; 
Wing, 1965; Harms et al., 1966; Kornegay, 1974; Adams, 1990).

O processamento do resíduo de panificação envolve a mistura dos materiais existentes (restos de bolos, pães e biscoitos), separação mecânica de substâncias indesejáveis, uma série de operações de padronização do tamanho das partículas, trituração ou moagem, desidratação até $6 \%$ de umidade e, posteriormente, obtenção de um produto final com consistência para ser analisado e incluído na formulação de rações animais (Wallace, 1965). Entretanto, Saleh et al. (1996) trabalharam com produto que apresentava um conteúdo de umidade de $10 \%$.

O resíduo de panificação é classificado como alimento energético, dado a sua alta concentração de carboidratos solúveis (Arosemena et al., 1995). Contudo, o uso de tal resíduo pode estar limitado em virtude do reduzido número de avaliações e do seu valor nutricional em animais (Harris Junior \& Staples, 1993).

Trabalhos relatam a existência de grandes diferenças na composição química dos resíduos de panificação atualmente utilizados na alimentação animal, uma vez que a magnitude das respostas sobre o desempenho - seja em ganho de peso, consumo de alimentos ou conversão alimentar -, depende da sua quantidade na ração total (Wallace, 1965; Harms et al., 1966; Waldroup et al., 1982; Dale et al., 1990; Arosemena et al., 1995; Hinders, 1995).

O resíduo de panificação pode ser excelente matéria-prima para rações de bovinos leiteiros, e é possível que a presença de ingredientes cozidos tragam outros benefícios para seu uso, como fonte de energia (Balch \& Rowland, 1957). Este resíduo foi avaliado como ingrediente para rações de vacas leiteiras (Wing, 1965; Adams, 1990), novilhos (Kirk \& Peacock, 1964, citados por Harms et al. 1966; Milton \& Brandt, 1993), ovinos (Champe \& Church, 1980) e búfalos (Spers, 1996). Segundo Harris Junior \& Staples (1993), o resíduo de panificação pode ser usado numa proporção de 20 a $25 \%$ do concentrado destinado à alimentação de ruminantes.

Adams (1990) relata que este resíduo pode ser usado até o máximo de $20 \%$ da matéria seca do concentrado, ou $10 \%$ da matéria seca da ração total para vacas leiteiras; entretanto, sua ingestão deveria ser limitada para vacas em lactação, dada a diminuição do teor de gordura no leite, em decorrência da alta porcentagem de carboidratos solúveis encontrada neste subproduto. Poderia até mesmo ser usado em maior proporção para alimentação de novilhas e vacas secas.

Kirk \& Peacock (1964), citados por Harms et al. (1966), afirmam que o resíduo de panificação pode substituir até $10 \%$ do milho nas rações de bezerros em confinamento, pois promove maior ganho de peso e melhora a eficiência alimentar em $4 \%$, comparado com o nível de $5 \%$ de resíduo de panificação na dieta.

Milton \& Brandt (1993) avaliaram o desempenho de novilhos de corte em terminação alimentados com rações contendo resíduo de panificação, substituindo o milho em $15 \%$ e $30 \%$ da matéria seca dietética, mantendo-se todas as rações isonitrogenadas. Neste estudo, a ingestão de matéria seca foi reduzida em $6,5 \%$ pela inclusão de $30 \%$ de resíduo de panificação, porém não foi observada diferença no ganho de peso diário ou eficiência alimentar entre os tratamentos.

Spers (1996), trabalhando com búfalos em crescimento, substituiu o milho por resíduo de panificação nas proporções de $33 \%$, 66\% e $100 \%$, encontrando melhora no ganho de peso diário até a proporção de $33 \%$ de substituição do milho pelo resíduo de panificação na dieta $(\mathrm{P}<0,01)$. Entretanto, as substituições de $66 \%$ e $100 \%$ do milho causaram redução no ganho de peso diário, e o autor sugere que este fato deva ser atribuído ao aumento na densidade energética das rações. A conversão alimentar piorou com as inclusões de resíduo, em todos os níveis estudados, e foram observados os valores de 8,78, 8,70, 10,93 e 13,90 , respectivamente, com relação às substituições de $0 \%, 33 \%, 66 \%$ e $100 \%$ do milho pelo resíduo de panificação na dieta. Houve melhora nos coeficientes de digestibilidade aparente da matéria seca com o aumento dos níveis de substituição do milho pelo resíduo de panificação nas $\operatorname{dietas}(\mathrm{P}<0,01)$.

Champe \& Church (1980), trabalhando com ovinos, realizaram um ensaio de digestibilidade dos resíduos de panificação comercializados, quando estes contribuíam com $20 \%$ ou $40 \%$ de uma dieta basal.

A digestibilidade de todos os componentes da dieta aumentou com a adição de resíduo de panificação $(\mathrm{P}<0,05)$, em ambos os níveis empregados, e observou-se um acréscimo marcante sobre a digestibilidade do extrato etéreo. Os coeficientes de 
digestibilidade do resíduo de panificação foram semelhantes, nos dois níveis empregados.

O objetivo deste trabalho foi o de estudar os efeitos da substituição parcial do milho pelo resíduo de panificação proveniente da fabricação de biscoitos, na dieta de novilhos da raça Holandesa.

\section{Material e Métodos}

$\mathrm{O}$ experimento foi desenvolvido na Faculdade de Medicina Veterinária e Zootecnia da Universidade de São Paulo, no Campus Administrativo de Pirassununga, no período de janeiro a junho de 1996. A instalação experimental utilizada foi um galpão coberto, com bebedouros comunitários de alvenaria e cochos individuais para alimentação, confeccionados em madeira.

$\mathrm{O}$ resíduo de panificação $(\mathrm{RP})$ utilizado neste estudo era constituído de sobras da fabricação de biscoitos provenientes de fábricas da região de Marília, SP, e apresentava a seguinte composição química, com base na matéria seca: $9,39 \%$ de proteína bruta $(\mathrm{PB}) ; 0,04 \%$ de fibra bruta (FB); 17,11\% de extrato etéreo (EE); 1,91\% de matéria mineral (MM); $0,075 \%$ de cálcio (Ca); $0,17 \%$ de fósforo (P) e 93,14\% de matéria seca (MS).

As dietas experimentais foram compostas de fubá de milho, farelo de trigo, farelo de soja, RP e feno de Coastcross (Cynodon dactylon L.), usado como único volumoso, picado em peneira de $4 \mathrm{~mm}$. Um suplemento mineral compôs $1 \%$ da mistura de concentrados. Os concentrados e o volumoso foram misturados e peletizados na forma de ração completa. As dietas permaneceram isonitrogenadas, mas o teor de extrato etéreo aumentou à medida que $\mathrm{o}$ milho foi substituído pelo resíduo. A Tabela 1 mostra a proporção dos ingredientes nas rações experimentais, com base na matéria seca. A Tabela 2 mostra a composição química das diferentes dietas experimentais, com base na matéria seca.

Os animais receberam, no período pré-experimental, dieta balanceada segundo as recomendações do National Research Council (1989); foi fornecido feno de Coast-cross integral como volumoso, na proporção de $30 \%$ da ração total. Esse período compreendeu 30 dias, destinados à adaptação dos animais ao manejo e instalações experimentais.

O experimento teve início com aproximadamente 60 dias após o desaleitamento, quando os animais apresentavam, em média, 123 dias de idade, e se estendeu por 120 dias.

No período experimental, a alimentação foi fornecida duas vezes ao dia, pela manhã ( $7 \mathrm{~h} 30$ às $8 \mathrm{~h} 30)$ e à tarde (13h às 14h), ficando a água disponível ad libitum.

Os animais foram provenientes de uma única propriedade, totalizando 20 bezerros machos da raça Holandesa, variedade preta-e-branca, puros por cruzamento, todos saudáveis, e que receberam quantidades adequadas de colostro materno.

Foram realizadas duas pesagens, no primeiro e segundo dias experimentais, utilizando o peso médio de cada animal para alocá-los em blocos.

Os animais foram submetidos a quatro tratamentos: ração controle com milho, sem resíduo de panificação; $10 \%$ de resíduo de panifícação substituindo o milho na mistura de concentrados; $20 \%$ de resíduo de panificação substituindo o milho na mistura de concentrados; $30 \%$ de resíduo de panificação substituindo o milho na mistura de concentrados.

O desempenho dos animais foi avaliado por meio do ganho de peso, altura na cernelha e perímetro torácico.

As pesagens foram realizadas a cada sete dias, sempre pela manhã, e com jejum hídrico e alimentar prévio de dose horas, sendo mensuradas no período da tarde a altura na cernelha e o perímetro torácico

Tabela 1. Composição porcentual das dietas com base na matéria seca.

\begin{tabular}{lrrrr}
\hline Ingredientes & \multicolumn{3}{c}{$\begin{array}{c}\text { Nível de inclusão de resíduo de } \\
\text { panificação no concentrado (\%) }\end{array}$} \\
\cline { 2 - 5 } & \multicolumn{1}{c}{0} & \multicolumn{1}{c}{10} & \multicolumn{1}{c}{20} & \multicolumn{1}{c}{30} \\
\hline Resíduo de panificação & 0,00 & 7,26 & 14,48 & 21,63 \\
Fubá de milho & 45,69 & 38,45 & 31,19 & 24,04 \\
Farelo de soja 46\% & 16,18 & 16,27 & 16,27 & 16,43 \\
Farelo de trigo & 6,99 & 6,97 & 6,97 & 6,95 \\
Suplemento mineral $^{(1)}$ & 0,78 & 0,78 & 0,78 & 0,78 \\
Feno de Coast-cross & 30,36 & 30,27 & 30,31 & 30,17 \\
\hline
\end{tabular}

(1) Suplemento mineral (níveis de garantia por $\mathrm{kg}$ do produto): fósforo, $80 \mathrm{~g}$, cálcio, $140 \mathrm{~g}$; sódio, $112 \mathrm{~g}$; magnésio, $25 \mathrm{~g}$; manganês, $1.260 \mathrm{mg}$; ferro, $1.300 \mathrm{mg}$; zinco, $2.520 \mathrm{mg}$; cobre, $1.500 \mathrm{mg}$; cobalto, $200 \mathrm{mg}$; iodo, $195 \mathrm{mg}$; selênio, $20 \mathrm{mg}$; flúor máximo, $800 \mathrm{mg}$; veículo q.s.p., $1.000 \mathrm{~g}$.

Tabela 2. Composição química das dietas experimentais, com base na matéria seca.

\begin{tabular}{lrrrr}
\hline Composição química & \multicolumn{3}{c}{$\begin{array}{l}\text { Nível de inclusão do resíduo de } \\
\text { panificação no concentrado }(\%)\end{array}$} \\
\cline { 2 - 5 } & \multicolumn{1}{c}{0} & \multicolumn{1}{c}{10} & \multicolumn{1}{c}{20} & \multicolumn{1}{c}{30} \\
\hline Matéria seca & 89,11 & 89,41 & 89,54 & 89,31 \\
Proteína bruta & 16,39 & 16,41 & 16,02 & 16,30 \\
Fibra bruta & 10,71 & 10,32 & 10,56 & 10,85 \\
Fibra em detergente neutro & 29,34 & 27,41 & 27,44 & 28,00 \\
Fibra em detergente ácido & 14,70 & 14,27 & 14,10 & 15,02 \\
Extrato etéreo & 2,37 & 3,14 & 3,60 & 4,61 \\
Matéria mineral & 4,23 & 3,99 & 4,21 & 3,80 \\
Cálcio & 0,43 & 0,37 & 0,43 & 0,36 \\
Fósforo & 0,40 & 0,37 & 0,38 & 0,33 \\
\hline
\end{tabular}


A altura na cernelha foi tomada a cada sete dias, utilizando-se uma barra de madeira de $1,5 \mathrm{~m}$ de altura à qual adaptou-se uma fita métrica. Eram tomadas três alturas no dia, sempre abordando o animal pelo lado esquerdo, sendo a média das três utilizada como valor semanal da altura

O perímetro torácico foi mensurado, utilizando-se fita métrica, colocada na região do cilhadouro, logo atrás dos membros anteriores do animal, e para este parâmetro foi tomada apenas uma medida semanalmente.

A intensidade da diarréia foi avaliada conforme Lucci (1989), classificando-a quanto à intensidade, usando notas de 1 a 4 . A duração da diarréia foi avaliada em porcentagem de dias que o animal teve suas fezes anormais, ou seja, com notas de 2 a 4

Foi ainda utilizado um índice de consistência fecal (ICF), unindo as duas variáveis, intensidade e duração, em um mesmo modelo matemático. Este valor foi obtido multiplicando-se o grau de consistência fecal pela sua duração em dias, indicando que, quanto maior o índice, mais intensa e duradoura foi a diarréia (Passini, 1997).

O delineamento experimental foi o de blocos casualizados (Pimentel-Gomes, 1985), com quatro tratamentos e cinco blocos, totalizando 20 animais Os resultados foram analisados por meio do programa computacional Statistical Analysis System (SAS Institute, 1985), sendo anteriormente verificada a normalidade dos resíduos pelo Teste de Shapiro-Wilk (Proc Univariate) e a homogeneidade das variâncias, comparadas pelo teste $\mathrm{F}$. Estando os dados dentro destas premissas básicas, passaram a ser submetidos à análise de variância através do procedimento General Linear Models (Proc GLM), utilizando regressão polinomial para os níveis de resíduos de panificação. Foi adotado um nível de significância de 5\% em todos os testes realizados.

\section{Resultados e Discussão}

Não houve diferença estatística significativa entre os tratamentos quanto às médias do ganho de peso diário, consumo de alimentos, conversão alimentar, altura na cernelha e perímetro torácico (Tabela 3).
Os resultados são condizentes com os obtidos por Milton \& Brandt (1993), em que a inclusão de 15\% ou $30 \%$ de resíduo de panificação seco em substituição ao milho nas dietas de garrotes cruzados, em fase de terminação, não alterou o ganho de peso diário ou a eficiência de conversão de alimentos, sugerindo, os autores, que o resíduo de panificação possui valor energético semelhante ao do grão de milho. Contudo, a ingestão de MS foi deprimida na ordem de $6,5 \%$, em relação ao grupo-controle, quando $30 \%$ de resíduo de panificação foi administrado; este efeito foi associado ao aumento do teor de gordura da dieta.

No presente experimento, não houve diferença estatisticamente significativa entre os tratamentos em relação à ingestão de alimentos, embora o teor de extrato etéreo das rações tenha aumentado progressivamente com as maiores taxas de substituição do milho pelo resíduo de panificação.

Desta forma, pode-se sugerir que a redução da ingestão de matéria seca observada por Milton \& Brandt (1993) poderia ser atribuída a outros fatores que não ao conteúdo de gordura da dieta, visto que os teores máximos de extrato etéreo foram próximos nos dois estudos: 5,1\% (Milton \& Brandt, 1993) e $4,6 \%$ no presente trabalho.

Spers (1996), trabalhando com búfalos, fez referência aos elevados teores de extrato etéreo presentes no resíduo de panificação. Em seu estudo, o autor reportou redução na média diária de ganho de peso quando o milho foi substituído por resíduo de panificação em $66 \%$ e $100 \%$, sugerindo, assim, que o aumento da densidade energética das rações tenha causado redução do consumo de alimentos, e, conseqüentemente, dos ganhos de peso. Porém, a inclusão de $33 \%$ de resíduo demonstrou efeito benéfico sobre o ganho de peso diário $(\mathrm{P}<0,01)$, embora alterando a ingestão de alimentos, visto que as conversões alimentares pioraram em todos os níveis de in-

Tabela 3. Médias diárias de ganho de peso, consumo de matéria seca, conversão alimentar, altura na cernelha e perímetros torácicos.

\begin{tabular}{|c|c|c|c|c|c|}
\hline \multirow[t]{2}{*}{ Variável } & \multicolumn{4}{|c|}{ Nível de inclusão do resíduo de panificação no concentrado (\%) } & \multirow{2}{*}{$\begin{array}{l}\mathrm{CV} \\
(\%)\end{array}$} \\
\hline & 0 & 10 & 20 & 30 & \\
\hline Ganho de peso (kg/dia) & 1,229 & 1,296 & 1,278 & 1,262 & 13,31 \\
\hline Consumo de matéria seca (kg/dia) & 5,842 & 6,133 & 6,644 & 6,268 & 10,61 \\
\hline Conversão alimentar & 4,796 & 4,763 & 5,233 & 4,998 & 8,56 \\
\hline Altura na cernelha $(\mathrm{cm})$ & 187,6 & 177,4 & 185,4 & 187,6 & 11,25 \\
\hline Perímetro torácico $(\mathrm{cm})$ & 347,0 & 379,0 & 379,0 & 363,0 & 9,56 \\
\hline
\end{tabular}


clusão do resíduo de panificação à dieta. Houve melhora no coeficiente de digestibilidade aparente da matéria seca com o aumento dos níveis de substituição do milho pelo resíduo de panificação nas dietas $(\mathrm{P}<0,01)$.

O aumento no consumo de alimentos poderia ser explicado por um aumento na apetibilidade das rações contendo resíduo de panificação, fator este já mencionado por Wallace (1965) e McConnell \& Williams Junior (196-) em seus estudos, sugerindo o uso do resíduo de panificação como ingrediente palatabilizante nas dietas de bezerros e leitões.

A maior ingestão de matéria seca em rações contendo resíduo de panificação, em relação às formuladas com ingredientes tradicionais, foi relatada por Combs et al. (1964) e Peo (1965) quando substituíram totalmente o soro de leite da dieta de leitões (20\%) pelo resíduo de panificação, e McConnell \& Williams Junior ([196-]) trabalhando com suínos em fase de creche, utilizando $10 \%$ de resíduo.

Alguns estudos mostraram haver melhora na conversão alimentar quando dietas formuladas com resíduo de panificação foram empregadas; isto, por causa da redução do consumo de alimentos ou melhora na eficiência alimentar (Wallace et al., 1964; Kirk \& Peacock, 1964 citados por Harms et al., 1966; Arrington, 1965; Peo, 1965; Potter et al., 1971; Kornegay, 1974).

Com relação à consistência das fezes dos animais, não houve diferença estatística significativa quanto à freqüência e intensidade de diarréia entre os tratamentos contendo $10 \%, 20 \%$ e $30 \%$ de resíduo de panificação em relação ao controle $(0 \%)$, com exceção da diarréia de grau 3 e do índice de consistência fecal, em que foi observado um desvio da curva quadrática $(\mathrm{P}<0,05)$ (Tabela 4). Esta observação pode

Tabela 4. Médias da incidência de diarréia (\%) e índice de consistência fecal (ICF) nos diferentes tratamentos.

\begin{tabular}{|c|c|c|c|c|c|}
\hline \multirow[t]{2}{*}{$\begin{array}{l}\text { Intensidade } \\
\text { de diarréia }\end{array}$} & \multicolumn{4}{|c|}{$\begin{array}{l}\text { Nível de inclusão do resíduo de } \\
\text { panificação no concentrado (\%) }\end{array}$} & \multirow[t]{2}{*}{$\begin{array}{l}\mathrm{CV} \\
(\%)\end{array}$} \\
\hline & 0 & 10 & 20 & 30 & \\
\hline Grau 1 & 67,33 & 73,33 & 48,17 & 70,50 & 29,86 \\
\hline Grau 2 & 29,00 & 24,33 & 40,50 & 24,50 & 52,54 \\
\hline Grau $3^{(1)}$ & 3,33 & 2,17 & 9,67 & 4,50 & 97,58 \\
\hline Grau 4 & 0,33 & 0,17 & 1,67 & 0,50 & 179,55 \\
\hline $\mathrm{ICF}^{(1)}$ & 34,17 & 32,29 & 41,21 & 33,75 & 17,41 \\
\hline
\end{tabular}

${ }^{(1)}$ Desvio da curva quadrática $(\mathrm{P}<0,05)$. ser atribuída ao acaso, e não possui explicação biológica. O uso do ICF reduziu os coeficientes de variação encontrados com relação aos diferentes graus de intensidade de diarréias.

O custo do quilo da ração diminuiu à medida que foi adicionado o resíduo à dieta; contudo, quanto maior o preço do milho, mais lucrativa torna-se a substituição deste pelo resíduo de panificação.

No presente experimento, o milho foi comprado a $\mathrm{R} \$ 0,14 / \mathrm{kg}$, e o resíduo foi adquirido por $\mathrm{R} \$ 0,05$. Neste caso, a inclusão de resíduo de panificação em substituição ao milho da dieta causou redução no custo do quilo de ração em 3,74\%, 7,44\% e 10,90\%, respectivamente nas dietas contendo $10 \%, 20 \%$ e $30 \%$ de resíduo, em relação à dieta-controle.

\section{Conclusões}

1. O resíduo de panificação pode substituir o milho das dietas de novilhos até a proporção de $30 \%$ na mistura de concentrados, sem causar prejuízos ao desempenho dos animais, seja quanto à ingestão de alimentos, ao ganho de peso, à conversão alimentar, seja à incidência de diarréias.

2. A utilização do resíduo de panificação como fonte alternativa de alimentação reduz os custos de produção de animais confinados.

\section{Agradecimentos}

Ao professor Richard Miles, da Universidade da Flórida, pelo envio de parte das referências bibliográficas deste estudo; ao professor Paulo Henrique Mazza Rodrigues, pelo auxílio com as análises estatísticas; aos técnicos do Laboratório de Bromatologia do Departamento de Nutrição e Produção Animal da FMVZ-USP; à Prefeitura do Campus Administrativo de Pirassununga, pela concessão da instalação experimental; à FAPESP, pela bolsa de estudo concedida e pelo auxílio financeiro para este trabalho.

\section{Referências}

ADAMS, R. S. Use of commodity ingredients and food processing wastes in the Northeast: dairy feeding systems. In: DAIRY FEEDING SYSTEMS SYMPOSIUM, 1990, Harrisburg. Proceedings... Harrisburg : Northeast Regional Agricultural Engineering Service, 1990. p. 176-183.

Pesq. agropec. bras., Brasília, v. 36, n. 4, p. 689-694, abr. 2001 
AROSEMENA, A.; DEPETERS, E. J.; FADEL, J. G Extent of variability in nutrient composition within selected by-product feedstuffs. Animal Feed Science and Technology, Amsterdam, v. 54, p. 103-120, 1995

ARRINGTON, L. R. Animal feeds from many sources dried bakery waste. Florida Agricultural Experiment Station Research Report, Gainesville, v. 10, n. 1, p. 8-9, 1965

BALCH, D. A.; ROWLAND, S. J. Volatile fatty acids and lactic acid in the rumen of dairy cows receiving a variety of diets. British Journal Nutrition, Wallingford, v. 2, p. $288-292,1957$.

CHAMPE, K. A; CHURCH, D. C. Digestibility of dried bakery product by sheep. Journal of Animal Science Champaign, v. 51, n. 1, p. 25-27, 1980.

COMBS, G. E.; WALLACE, H. D.; BERRY, T. H. Dried bakery product as a replacement for dried skim milk in starter rations. Gainesville : Florida Agricultural Experiment Station, 1964. p. 1-4. (Animal Science Department Mimeograph Report, AN65-1).

DALE, N. M.; PESTI, G. M.; ROGERS, S. R. True metabolizable energy of dried bakery product. Poultry Science, Champaign, v. 69, p. 72-75, 1990.

HARMS, R. H.; DAMRON, B. L.; WALDROUP, P. W Dried bakery product as an ingredient for poultry feeds. Feedstuffs, Minnetonka, v. 38, n. 42, p. 42-43, 1966

HARRIS JUNIOR, B.; STAPLES, C. R. Feeding byproduct feedstuffs to dairy cattle. Gainesville : University of Florida, 1993. p. 1-6

HINDERS, R. Cornell system useful in evaluation of rations containing byproducts. Feedstuffs, Minnetonka, v. 67, n. 13 , p. 12 , Nov. 1995

KORNEGAY, E. T. Blended dried bakery product for growing and finishing swine. Feedstuffs, Minnetonka, v. 46, n. 15 , p. 23,1974

LUCCI, C. S. Bovinos leiteiros jovens: nutrição, manejo, doenças. São Paulo : Nobel/EDUSP, 1989. 371 p

McCONNELL, J. C.; WILLIAMS JUNIOR, W. P. Cookie meal for young pigs. Cold Spring : Griffin Sales, Bakery Feeds/Clemson University-Food Science Department, [196-]. p. 13

MILTON, C. T.; BRANDT, R. T. Utilization of dried bakery product by finishing beef steers. Cattlemen's Day, Kentucky, v. 1, p. 104-106, 1993
NATIONAL RESEARCH COUNCIL (Washington, Estados Unidos). Nutrient requirements of dairy cattle. Washington : National Academy Press, 1989. $157 \mathrm{p}$

PASSINI, R. Efeito da substituição parcial do milho da dieta pelo resíduo de panificação sobre o desempenho e características da carcaça de novilhos da raça Holandesa. Pirassununga : USP, 1997. 67 p. Dissertação de Mestrado.

PEO, E. R. Bakery product fed as skim milk replacement Feedstuffs, Minnetonka, v. 37, n. 12, p. 9, 1965.

PIMENTEL-GOMES, F. Curso de Estatística Experimental. Piracicaba : ESALQ, 1985. 162 p.

POTTER, L. M.; SHELTON, J. R.; KELLY, M. Effects of zinc bacitracin, dried bakery product and different fish meals in diets of young turkeys. Poultry Science, Champaign, v. 50, p. 1109-1115, 1971

SALEH, E. A.; WATKINS, S. E.; WALDROUP, P. W High-level usage of dried bakery product in broiler diets. Journal of Applied Poultry Research, Fayettesville, v. 5, p. $33-38,1996$

SAS INSTITUTE (Cary, Estados Unidos). Statistical analysis system. 5. ed. Cary, 1985. 1686 p.

SPERS, R. C. Efeito da substituição do milho e do farelo de soja pelo resíduo de panificação no desempenho de bubalinos em crescimento. Botucatu : UNESP, 1996 27 p. Dissertação de Mestrado

WALDROUP, P. W.; WHELCHEL, D. L.; JOHNSON, Z. B. Variation in nutrient content of samples of dried bakery product. Animal Feed Science and Technology, Amsterdam, n. 7, p. 419-421, 1982.

WALLACE, H. D. Dried bakery product as a feed for swine. Feedstuffs, Minnetonka, v. 37, n. 25, p. 52-53, 57 , 1965

WALLACE, H. D.; COMBS, G. E.; CONNESS, R. G. Dried bakery product as a substitute for corn and as a source of B-vitamins for the growing-finishing pig. Gainesville : Florida Agricultural Experiment Station, 1964. p. 1-6. (Animal Science Department Mimeograph Report, AN64-15)

WING, J. M. Preliminary evaluation of dried bakery product in dairy cattle rations. Feedstuffs, Minnetonka, v. 37, n. 19 , p. $38,128,1965$ 